\title{
Literatura qualificada sobre capacidade absortiva para inovação em NEBTs e startups
}

* Bruno Alencar Pereira

Universidade de Brasília (UnB), Brasília (DF), Brasil

E-mail: alenbruno@gmail.com

* Josivania Silva Farias

Universidade de Brasília (UnB), Brasília (DF), Brasil

E-mail: josivania@unb.br

\section{Resumo}

Este trabalho consiste em revisar e sistematizar a literatura relativa à Capacidade Absortiva (ACAP) para inovação em novas empresas de base tecnológica (NEBTs) e startups. Adicionalmente, o estudo identifica temas, abordagens teóricas e fatores de desenvolvimento da ACAP para inovação nestes empreendimentos, promovendo um panorama deste fenômeno. A pesquisa analisa 103 artigos selecionados nas bases de periódicos Emerald, Science Direct, Ebsco (Academic Search Premier), Web of Science, Scopus e Springerlink. Identificou-se a tendência para pesquisas empíricas baseadas em métodos quantitativos com utilização de surveys e técnicas de análise fundamentadas em estatística inferencial. As quatro categorias mais investigadas dizem respeito à relação entre a ACAP e redes, antecedentes e potencial de realização, exploração de fatores/recursos internos ou externos, e estratégias de inovação. Os resultados ainda possibilitaram a proposição de lacunas e direcionamentos como contribuição para futuras agendas.

Palavras-chave: Capacidade Absortiva; Inovação; Novas empresas de base tecnológica; Startups. 


\section{Qualified literature on absorptive capacity for innovation in NTBFs and startups}

\section{Abstract}

This study consists of reviewing and systematizing the literature on Absorptive Capacity (ACAP) for innovation in new technology-based firms (NEBTs) and startups. In addition, the study identifies themes, theoretical approaches and factors for ACAP and innovation development into these ventures; providing an overview of this phenomenon. The research analyzes 103 articles selected from databases such as Emerald, Science Direct, Ebsco (Academic Search Premier), Web of Science, Scopus and Springerlink. Most of studies identified relates to empirical research based on quantitative methods; using surveys and analysis techniques based on inferential statistics. The four prominent categories concern to the relationship between ACAP and networks, antecedents and potential of ACAP, exploration of internal or external factors/resources, and innovation strategies. The results also enable the proposition of gaps and directions as a contribution to future agendas.

KeYwords | Absorptive Capacity; Innovation; New technology-based firms; Startups 


\section{Introdução}

A capacidade absortiva é crucial para a criação de inovaçóes e tecnologias que proporcionam vantagem competitiva através da entrega de novas soluçóes ao mercado. Segundo Zheng, Liu e George (2010), há evidências que demonstram que a capacidade inovadora está altamente correlacionada com o potencial de crescimento e desempenho de novas empresas de base tecnológica (NEBTs) e startups. Porém, tais evidências ainda não suprem sistematicamente a compreensão sobre a relação entre a absorção de conhecimento e inovação nestes empreendimentos. Neste sentido, um olhar especial se torna necessário no entendimento sobre essa relação e no seu efeito sobre as práticas organizacionais destes empreendimentos (ROSSETTO et al., 2019).

Cohen e Levinthal $(1989,1990)$ iniciam a abordagem conceitual sobre a Absorptive Capacity (ACAP), ou Capacidade Absortiva em português, que é considerada como as habilidades e conhecimentos de um empreendimento, que possibilitam o reconhecimento do valor de uma nova informação, ideia ou insight, para assimilação e aplicação mercadológica, como fator crítico para suas capacidades de inovação.

Para compreensão e enquadramento do que se tratam as novas empresas de base tecnológica (NEBTs), levaram-se em consideração conceituações indicadas pelos autores Storey e Tether (1998) e Arthur D. Little Ltd. (1977), que as definem como empresas de propriedade independente, estabelecidas por não mais que 25 anos, que atuam na exploração de uma invençãa ou inovação tecnológica. Estes empreendimentos ainda possuem como característica a orientaçáo para riscos tecnológicos substanciais, abrangendo setores que podem ser vistos como intensivos em tecnologia.

Da mesma forma, para melhor delineamento do termo startup, levaram-se em consideração conceituaçóes indicadas por Ries (2011) e Blank e Dorff (2012). Esses autores definem startup como uma organização de caráter temporário, sob condiçóes de alta incerteza, que busca um modelo de negócio rentável a partir do desenvolvimento de uma ideia inovadora. A sutil distinção teórico-prática entre NEBTs e startups é observada quanto ao foco no crescimento escalável e inovação baseada em modelo de negócio, elementos característicos das startups, nem sempre perceptíveis nas NEBTs.

Sem delimitação de ano inicial, esta revisão da literatura analisa 103 artigos publicados até dezembro de 2017, em língua inglesa, para a compreensão e avanços da ACAP com foco em NEBTs e startups. Foram utilizados aspectos descritivos e 
exploratórios de uma abordagem qualitativa e quantitativa para a identificação do panorama dos estudos relacionados. Incluem-se temas e abordagens da ACAP, culminando na identificação de fatores que influenciam o seu desenvolvimento para inovação nesses empreendimentos. Por fim, lacunas são identificadas e direcionamentos são indicados para pesquisas que possam aprofundar a evolução do tema.

\section{Referencial teórico}

\subsection{Capacidade Absortiva (ACAP)}

O desenvolvimento da ACAP e o desempenho inovador são dependentes da história ou do caminho estrategicamente escolhido pelas organizaçóes. Empresas são sensíveis às características de aprendizagem no ambiente em que operam, em que a ACAP é desenvolvida pelas rotinas que criam capacidades dinâmicas para operação e para exploração conjunta de conhecimentos existentes ou externos (COHEN; LEVINTHAL, 1990; EISENHARDT; MARTIN, 2000).

Seguindo a evolução da ACAP, Zahra e George (2002) sugerem que a capacidade absortiva é composta por dois subconjuntos de capacidades absortivas, as "potenciais" e as "realizadas". A Capacidade Absortiva Potencial ou Potential Absorptive Capacity (PACAP) engloba as capacidades de aquisição e assimilação de conhecimento, ao passo que a Capacidade Absortiva Realizada ou Realized Absorptive Capacity (RACAP) corresponde à transformação e exploração do conhecimento. Relevantes estudos empíricos, como os de Fosfuri e Tribó (2008) e Ali e Park (2016), também reforçam a ocorrência destes subconjuntos.

Depois de 20 anos da construção seminal do conceito por Cohen e Levinthal, Lewin, Massini e Peeters (2011) traçaram um panorama sobre a ACAP. Esses autores identificaram vasta literatura sobre o constructo e pesquisas em áreas diversas, incluindo a teoria da organização, gestão estratégica e economia. Também constataram que os processos que constituem a ACAP continuam sendo uma caixa preta. Portanto, equilibrar processos internos de criação de conhecimento com a identificação, aquisição e assimilação de novos conhecimentos ainda precisam ser mais bem compreendidos.

Observando a literatura, identifica-se uma relevante e crescente ênfase em processos relacionados à ACAP. Estudos sobre este constructo desenvolveram links estreitos para dois fluxos no campo teórico estratégico e organizacional. Por um lado, uma literatura sobre transferência de conhecimento interorganizacional e 
aprendizado e, por outro lado, a literatura sobre capacidades dinâmicas com foco na vantagem competitiva sustentada através de processos de aprendizagem e mudança (GEBAUER; WORCH, 2015).

\subsection{Capacidade absortiva em novas empresas de base tecnológica (NEBTs) e startups}

Por sua orientação empreendedora, as NEBTs e startups parecem possuir características «genéticas» que facilitam a detecção, absorção e exploração de conhecimento (COLOMBO; PIVA, 2008; COLOMBO; D'ADDA; PIVA, 2010). Entretanto, diferentemente de empresas tradicionais consolidadas, esses empreendimentos enfrentam e carregam riscos associados à escassez de recursos e conhecimentos complementares. Neste sentido, a ACAP possibilita a estas organizaçôes atributos que suprem tais necessidades levando a uma maior taxa sobrevivência e sucesso ao gerar inovaçôes (HYYTINEN; PAJARINEN; ROUVINEN, 2015).

Evidências na literatura indicam especificidades inerentes a estes empreendimentos, como por exemplo, que seus fundadores enfrentam os desafios do desenvolvimento tecnológico incerto, necessitando de flexibilidade estratégica para se adaptar e sobreviver em ambientes em mudança (ROBERTS; MEYER, 1991; ANDRIES; DEBACKERE, 2007; CANDI; VAN DEN ENDE; GEMSER, 2013; SAEMUNDSSON; CANDI, 2017). A literatura também sugere que o capital humano e o capital social sejam fatores essenciais para obtenção de maior capacidade absortiva, constituindo componentes primordiais para a ocorrência da ACAP nestes empreendimentos (DE JONG; FREEL, 2010, PRESUTTI; BOARI; MAJOCCHI, 2011; SALISU; ABU BAKAR, 2019).

Capacidades baseadas em recursos, rotinas e especialmente as capacidades dinâmicas têm impacto maior sobre a vantagem competitiva destes empreendimentos do que outros ativos intangíveis e tangíveis (EISENHARDT; MARTIN, 2000; PARADKAR; KNIGHT; HANSEN, 2015). A rede de contatos pode potencializar tais capacidades bem como a absorção de conhecimento e obtenção de recursos, influenciando positivamente a criação de inovações (ZHENG; LIU; GEORGE, 2010; SALISU; ABU BAKAR, 2019). Isto ocorre principalmente, nas startups, em que os gastos limitados com P\&D podem fazer com que estes empreendimentos sejam mais dependentes de alianças externas e interação frequente com parceiros diversos (DE JONG; FREEL, 2010; PRESUTTI; BOARI. MAJOCCHI, 2011). 
Como síntese das tipologias e especificidades dos empreendimentos abordados no estudo, é possível indicar que as NEBTs possuem inovaçáo focada no desenvolvimento tecnológico de produtos, direcionando recursos relevantes para o uso intenso de P\&D. Elas também se caracterizam por englobar empresas formalizadas e com maior tempo de existência, e com adoção de estruturas menos flexíveis do que as startups para escalabilidade mercadológica. Por sua vez, as startups possuem inovação diversificada entre produtos, serviços e modelo de negócio, com limitação de recursos para P\&D. Elas também se caracterizam por englobar organizaçóes formalizadas ou não em fase inicial de desenvolvimento, e com estrutura organizacional mais enxuta, possibilitando maior escalabilidade mercadológica.

\section{Metodologia}

Os autores conduziram o levantamento da literatura para a revisão durante o período de junho a dezembro de 2017, utilizando como fontes as bases obtidas no portal Periódicos da Capes (Coordenação de Aperfeiçoamento de Pessoal de Nível Superior), como Emerald, Science Direct, Ebsco (Academic Search Premier), Web of Science, Scopus e Springerlink.

Para a realização das buscas nos campos de título, ou resumo ou palavras-chave, foi utilizada, em única instância, a combinaçâo dos seguintes termos com operadores booleanos: "absorptive capacity ou absorption capacity" e "innovation" e "startup ou start-up ou new technology-based firms ou based technology ou technology companies ou technology firms", no sentido de se obter maior especificidade sobre o tema.

O uso dos termos technology companies ou technology firms foi incorporado na busca, o que abrangeu o escopo de empresas inovadoras investigadas, mantendo-se ainda o espectro de atuação característico principalmente às NEBTs. Por exemplo, Fosfuri e Tribo (2008) definem a amostra de 2.464 empresas inovadoras da Espanha, de propriedade independente e perfil pautado no uso intenso de tecnologia, porém, sem definiçấo de que sejam estabelecidas por não mais que 25 anos.

Sem delimitação do ano inicial, no sentido de se obter um levantamento amplo da literatura existente até dezembro de 2017, foram encontrados inicialmente 463 artigos. Como critério, os artigos encontravam-se em língua inglesa, relacionados a disciplinas pré-selecionadas nos campos de administração, negócios e gestão pela plataforma Periódicos da Capes. Durante o processo de seleção, os autores conduziram minuciosa análise observando resumos, introdução e métodos, com o intuito de identificar o enquadramento do estudo e tipologias dos empreendimentos abordados. 
Assuntos difusos não focalizando ACAP no desenvolvimento de empreendimentos ou inovaçóes foram retirados da base, restando 142 artigos. Como exemplos de estudos náo considerados têm-se Nätti, Hurmelinna-Laukkanen e Johnston (2014) investigando ACAP de redes de serviços, Deschesnes, Drouin e Couturier (2013) que pesquisaram a capacidade de absorção de escolas para inovar na promoção da saúde, ou Fertő, Molnár e Tóth (2016) que analisaram contextos de Inovação Aberta da cadeia alimentar da Hungria.

Seguindo critérios, dentre os 142 artigos, foram considerados apenas os estudos publicados em periódicos com fator de impacto igual ou superior a 1. A identificação do fator de impacto foi realizada pela base InCities Journal Citation Reports (JCR) acessada pelo portal Periódicos da Capes, sendo todos classificados no estrato A1 pelo sistema Qualis daquele ano (2017). Esse critério foi previamente adotado com vistas a assegurar uma maior relevância e qualidade do corpus de textos analisado. Após esse filtro, foram desconsiderados 30 artigos sem classificação no estrato A1. Também se observou que 9 artigos elegíveis se repetiam em uma ou mais bases, obtendo-se, portanto, 103 artigos para a análise final. Ressalta-se que, no período observado, em certas áreas como economia, turismo e outros, os mesmos periódicos apresentavam extrato Qualis inferior a A1.

Dos 103 artigos selecionados observou-se que 77 abordavam NEBTs, 15 abordavam startups, e 11 abordavam contexto teórico sobre ACAP e desenvolvimento inovador, mesmo quando não declarada explicitamente a especificidade de empreendimentos.

Os artigos selecionados foram organizados com o uso do software Mendeley Desktop 1.17.11, tendo seu conteúdo lido e analisado para a posterior categorização das informaçôes. Posteriormente, um arquivo no formato .xml foi exportado para o uso em planilha do software Microsoft Excel. A planilha foi utilizada como suporte para a análise quantitativa das informaçóes, contendo colunas como país de origem do artigo, ano, nome da revista, database de origem, fator de impacto, foco temático e aspectos metodológicos como natureza da pesquisa, técnicas de coleta e de análise.

Inicialmente, para a análise dos resultados, foram adotados aspectos quantitativos e descritivos para a identificação do panorama dos estudos relacionados. Em seguida, utilizou-se uma perspectiva qualitativa e exploratória para identificação, categorização e discussão das abordagens e fatores relacionados à ACAP. Por fim, são indicados os direcionamentos e lacunas existentes para pesquisas que possam aprofundar a evolução do tema. 


\section{Resultados}

\subsection{Aspectos bibliométricos}

Com a realização da revisão da literatura, observou-se que os artigos selecionados são oriundos de 47 periódicos diferentes com os respectivos fatores de impacto e número de artigos vinculados (ver Quadro 1). Ressalta-se que 9 artigos se repetiam entre as diferentes bases.

\section{QUADRO 1}

Fator de impacto e número de artigos vinculados por periódico

\begin{tabular}{|lcc|}
\hline \multicolumn{1}{|c}{ Periódico } & $\begin{array}{c}\text { Fator de Impacto } \\
\text { (InCities JCR) }\end{array}$ & $\begin{array}{c}\text { № de artigos } \\
\text { vinculados }\end{array}$ \\
\hline Research Policy & 4.66 & 13 \\
Journal of Business Research & 2.50 & 8 \\
Technological Forecasting and Social Change & 3.13 & 6 \\
Journal of Knowledge Management & 2.55 & 5 \\
Management Decision & 1.52 & 5 \\
Industrial Marketing Management & 3.67 & 4 \\
Technovation & 4.80 & 4 \\
European Management Journal & 2.36 & 3 \\
International Small Business Journal & 3.90 & 3 \\
Knowledge Management Research \& Practice & 1.48 & 3 \\
Strategic Management Journal & 5.48 & 3 \\
Technology Analysis \& Strategic Management & 1.49 & 3 \\
The Journal of Technology Transfer & 2.93 & 3 \\
European Journal of Innovation Management & 1.38 & 2 \\
IEEE Transactions on Engineering Management & 1.41 & 2 \\
Journal of Business \& Industrial Marketing & 1.83 & 2 \\
Journal of Business Venturing & 6.00 & 2 \\
Journal of Engineering and Technology Management & 2.68 & 2 \\
The Journal of High Technology Management Research & 1.47 & 2 \\
Asian Business \& Management & 1.17 & 1 \\
Asia Pacific Journal of Management & 2.47 & 2 \\
Creativity and Innovation Management & 1.55 & 2 \\
Entrepreneurship and Regional Development & 2.79 & 1 \\
European Journal of Marketing & 1.49 & 2 \\
\hline
\end{tabular}


QUADRO 1

Fator de impacto e número de artigos vinculados por periódico

\begin{tabular}{|lcc|}
\hline \multicolumn{1}{|c}{ Periódico } & $\begin{array}{c}\text { Fator de Impacto } \\
\text { (InCities JCR) }\end{array}$ & $\begin{array}{c}\text { № de artigos } \\
\text { vinculados }\end{array}$ \\
\hline Growth \& Change & 1.19 & 1 \\
IEEE Transactions on Professional Communication & 1.143 & 1 \\
Information and Organization & 1.85 & 1 \\
Industrial and Corporate Change & 2.19 & 1 \\
Industrial Management \& Data Systems & 2.94 & 1 \\
International Entrepreneurship and Management Journal & 2.40 & 1 \\
International Journal of Technology Management & 1.60 & 1 \\
International Journal of Operations \& Production & 2.95 & 1 \\
Management & 1.21 & 1 \\
Journal of Business-to-Business Marketing & 2.20 & 1 \\
Journal of Construction Engineering \& Management & 2.48 & 1 \\
Journal of Enterprise Information Management & 6.19 & 1 \\
Journal of International Business Studies & 2.19 & 1 \\
Journal of Manufacturing Technology Management & 3.22 & 1 \\
Long Range Planning & 1.28 & 1 \\
Management Research Review & 1.28 & 1 \\
Marketing \& Management of Innovations & 1.05 & 1 \\
Measuring Business Excellence & 3.02 & 1 \\
Organization Science & 4.31 & 1 \\
Omega & 2.87 & 1 \\
Small Business Economics & 1.17 & 1 \\
Strategic Change in Entrepreneurial Finance & 3.48 & 1 \\
Strategic Entrepreneurship Journal & 1.65 & 1 \\
The Learning Organization & & 1 \\
\hline
\end{tabular}

Fonte: Elaboração dos autores.

Observa-se no Quadro 1 que o periódico Research Policy e o Journal of Business Research se destacam com o maior número de publicaçóes sobre o tema. Nas bases pesquisadas, os estudos focalizados na ACAP direcionada para inovação em NEBTs ou startups tiveram sua primeira referência no ano de 1999. O primeiro estudo a abordar este contexto foi o artigo "Make and buy in innovation strategies: evidence from Belgian manufacturing firms", publicado por Veugelers no periódico Research Policy. 
O número de estudos relacionados a essa temática se manteve estável até o ano de 2009, com média anual de dois artigos e a expansão deste tema, em publicaçóes, ocorreu a partir do ano de 2010. Destaca-se o aumento significativo de publicaçóes nos anos de 2015 (13 artigos), 2016 (14 artigos) e 2017 (21 artigos), demonstrando a ascensão da agenda de pesquisas sobre o tema.

Em uma análise mundial de pesquisas relacionadas ao tema, observou-se que os autores e coautores que publicaram sobre o assunto possuem origem e vínculo institucional em 32 países diferentes. Dentre os países com maior número de publicaçóes vinculadas se destacam os Estados Unidos com 21 artigos, a Espanha com 18, a China e a Inglaterra com 15 cada, e a Alemaha e a Holanda com 7 cada. O Brasil possui 1 artigo publicado em 2017, "Intellectual capital, absorptive capacity and product innovation”, dos autores Engelman, Fracasso, Schmidt e Zen, pela revista Management Decision.

Quanto ao enquadramento, natureza e aspectos metodológicos dos artigos encontrados, 11,65\% (12 artigos), possuindo forte abordagem teórica, que foram catalogados como ensaios, revisão da literatura ou modelos conceituais, e 88,35\% (91 artigos) como artigos téorico-empíricos.

No que se refere à natureza dos 91 artigos teórico-empíricos, há uma predominância de estudos quantitativos (74 artigos) representando 71,84\% do total. Foram encontrados 11 artigos de natureza qualitativa, significando 10,68\% do total e 6 artigos de natureza híbrida (qualitativa e quantitativa) correspondendo a 5,83\% do total. Quanto aos métodos e técnicas de coleta de dados empregados nestes estudos, verificou-se que os questionários foram predominantes, sendo utilizados em 58 artigos, seguidos pelo uso de dados secundários em 26 artigos. Ressalta-se que o tipo de técnica de coleta de dados indicado nesta análise tratam-se do principal método utilizado em cada artigo, mesmo considerando que alguns deles tenham empregado outros métodos secundários. Exemplo: artigos que, primariamente, utilizaram survey online como coleta principal e, de forma complementar ou secundária, conduziram também entrevistas por telefone/pessoalmente para reforçar os achados.

Quanto ao uso de técnicas de análise utilizadas nos 11 artigos qualitativos, observou-se a predominância de entrevistas em 5 artigos, métodos mistos por entrevista e análise documental em 4 artigos, e apenas análise documental em 2 artigos. Tratando-se das técnicas de análise nos artigos quantitavivos e quali-quantitativos, foi percebida a predominânica do uso de estatística inferencial em 79 artigos, representando $76,7 \%$ do total dos estudos. 
Dentre os estudos quantitativos, destacam-se em maior número o uso de técnicas como regressão por modelagem de equação estrutural e mínimos quadrados em 22 artigos, modelos para variáveis dependentes binárias (logit/probit) em 16 artigos, regressão múltipla em 13 artigos, regressão binomial negativa ou para dados de contagem em 11 artigos, e análise fatorial e teste de confiabilidade de construtos latentes em 11 artigos. As diversas técnicas inferenciais adotadas podem ser vistas no Quadro 2.

QUADRO 2

Técnicas de análise baseadas em estatística inferencial nos estudos quantitativos

\begin{tabular}{|c|c|c|c|}
\hline Técnica(s) & Tipos & Qtd. & Autor(es) \\
\hline \multirow{6}{*}{ Regressão } & $\begin{array}{l}\text { Modelagem } \\
\text { de equação } \\
\text { estrutural } \\
\text { e mínimos } \\
\text { quadrados }\end{array}$ & 22 & $\begin{array}{l}\text { Chen, Lin e Chang (2009); Fernhaber e Patel (2012); García- } \\
\text { Morales, Bolívar-Ramos e Martín-Rojas (2014); Hughes et al. } \\
\text { (2014); Engelman et al. (2017); Shoham et al. (2017); Yao e } \\
\text { Chang (2017); Martínez-Cañas, Sáez Martínez e Ruiz Palomino } \\
\text { (2012); Berghman et al., (2013); Ferreras-Méndez et al. (2015); } \\
\text { Ali e Park (2016); Sheng e Chien (2016); Chitsaz, Liang e } \\
\text { Khoshsoroor (2017); Rothaermel e Thursby (2005); Fosfuri e } \\
\text { Tribó (2008); Benson e Ziedonis (2009); Zheng, Liu e George } \\
\text { (2010); Petti e Zang (2013); Cruz-González, López-Sáez e } \\
\text { Navas-López (2015); Xu (2016); Sears (2017); Wang, Guo e Yin } \\
\text { (2017) }\end{array}$ \\
\hline & $\begin{array}{l}\text { Modelos para } \\
\text { variáveis } \\
\text { dependentes } \\
\text { binárias (logit/ } \\
\text { probit) }\end{array}$ & 16 & $\begin{array}{l}\text { Veugelers e Cassiman (1999); Rothaermel e Thursby (2005); Rhee } \\
\text { (2008); Vega-Jurado et al. (2008); Hyytinen, Pajarinen e Rouvinen } \\
\text { (2015); Li, Youtie e Shapira et al. (2015); Aniruddha e Mital (2016); } \\
\text { Pereira e Leitão (2016); Agostini e Nosella (2017); Stulova e Rungi } \\
\text { (2017); Fosfuri e Tribó (2008); Faria, Lima e Santos (2010); Harison } \\
\text { e Koski (2010); Coeurderoy et al. (2012); Garcia Martinez, Zouaghi } \\
\text { e Sanchez Garcia (2017); Fukugawa (2013) }\end{array}$ \\
\hline & Múltipla & 13 & $\begin{array}{l}\text { Fosfuri e Tribó (2008); Wang e Han (2011); Serrano-Bedia, } \\
\text { López-Fernández e García-Piqueres (2012); Awang, Hussain e } \\
\text { Malek (2013); Liefner, Wei e Zeng (2013); Tavani, Sharifi, e } \\
\text { Ismail (2013); Lai e Weng (2014); Van Geenhuizen e Ye (2014); } \\
\text { Martinez-Senra et al. (2015); Díez-Vial e Montoro-Sánchez } \\
\text { (2016); Flor, Cooper e Oltra (2017); Gölgeci, Swiatowiec- } \\
\text { Szczepanska e Raczkowski (2017); Hughes et al (2017) }\end{array}$ \\
\hline & $\begin{array}{l}\text { Binomial } \\
\text { negativa ou } \\
\text { para dados de } \\
\text { contagem }\end{array}$ & 11 & $\begin{array}{l}\text { Nooteboom et al. (2007); Sugheir, Phan e Hasan (2012); } \\
\text { Nambisan (2013); Huang et al. (2015); Li, Youtie e Shapira } \\
\text { (2015); Aniruddha e Mital (2016); Wang et al., (2016); Agostini } \\
\text { e Nosella (2017); Huang (2017); Wang et al. (2016) }\end{array}$ \\
\hline & Hierárquica & 8 & $\begin{array}{l}\text { Jantunen (2005); Fosfuri e Tribó (2008); Cheng e Chen (2013); } \\
\text { Burcharth, Lettl e Ulhøi (2015); Cruz-González, López-Sáez } \\
\text { e Navas-López (2015); Parida, Oghazi e Cedergren (2016); } \\
\text { García-Sánchez, García-Morales e Martín-Rojas (2017); } \\
\text { Saemundsson e Candi (2017) }\end{array}$ \\
\hline & Poisson & 5 & $\begin{array}{l}\text { Nooteboom et al. (2007); Lee, Kim e Jang (2015); Wang et al. } \\
\text { (2016); Garcia Martinez, Zouaghi e Sanchez (2017); Huang } \\
\text { (2017) }\end{array}$ \\
\hline
\end{tabular}


QUADRO 2

Técnicas de análise baseadas em estatística inferencial nos estudos quantitativos

\begin{tabular}{|c|c|c|c|}
\hline Técnica(s) & Tipos & Qtd. & Autor(es) \\
\hline \multirow{8}{*}{ Regressão } & Moderada & 3 & $\begin{array}{l}\text { Zhou e Li (2012); Cheng e Chen (2013); Yoo, Sawyerr e Tan } \\
\text { (2015) }\end{array}$ \\
\hline & Linear & 2 & Deeds (2001); Hurmelinna-Laukkanen (2012) \\
\hline & Multinível & 1 & De Jong e Freel (2010) \\
\hline & Agrupada & 1 & Mueller (2007) \\
\hline & $\begin{array}{l}\text { Mínima } \\
\text { ordinária }\end{array}$ & 1 & Huang (2017) \\
\hline & $\begin{array}{l}\text { Método dos } \\
\text { momentos } \\
\text { generalizados }\end{array}$ & 1 & Filatotchev et al. (2011) \\
\hline & $\begin{array}{l}\text { Equação de } \\
\text { estimativa } \\
\text { generalizada }\end{array}$ & 1 & Luo, Lui e Kim (2017) \\
\hline & $\begin{array}{l}\text { Modelo de } \\
\text { estimação com } \\
\text { double log form }\end{array}$ & 1 & Negassi (2004) \\
\hline \multirow{5}{*}{ Diversos } & $\begin{array}{l}\text { Análise fatorial } \\
\text { e teste de } \\
\text { confiabilidade } \\
\text { de construtos } \\
\text { latentes }\end{array}$ & 11 & $\begin{array}{l}\text { Fosfuri e Tribó (2008); Vega-Jurado et al. (2008); Camisón } \\
\text { e Forés (2010); Flatten et al. (2011); Cheng e Chen (2013); } \\
\text { Martín-de Castro et al. (2013); Tavani, Sharifi e Ismail (2013); } \\
\text { García-Morales, Bolívar-Ramos e Martín-Rojas (2014); Yoo, } \\
\text { Sawyerr e Tan (2015); Larrañeta, Galán González e Aguilar } \\
\text { (2017); Chan, Oerlemans e Pretorius (2010) }\end{array}$ \\
\hline & $\begin{array}{l}\text { Análise } \\
\text { sociométrica } \\
\text { de redes sociais }\end{array}$ & 2 & $\begin{array}{l}\text { Díez-Vial e Montoro-Sánchez (2016); Martin-Rios e Erhardt } \\
\text { (2016) }\end{array}$ \\
\hline & $\begin{array}{l}\text { Modelo de } \\
\text { análise de } \\
\text { classes latentes }\end{array}$ & 1 & Grimpe e Sofka (2009) \\
\hline & $\begin{array}{l}\text { Teoria dos } \\
\text { conjuntos } \\
\text { aproximados }\end{array}$ & 1 & Van Geenhuizen e Nijkamp (2012) \\
\hline & $\begin{array}{l}\text { Análise } \\
\text { multivariada } \\
\text { de covariância } \\
\text { e variância }\end{array}$ & 1 & Larrañeta, Galán González e Aguilar (2017) \\
\hline \multirow{2}{*}{$\begin{array}{l}\text { Testes não } \\
\text { paramétricos }\end{array}$} & $\begin{array}{l}\text { Mann- } \\
\text { Whitney }\end{array}$ & 1 & Larrañeta, Galán González e Aguilar (2017) \\
\hline & $\begin{array}{l}\text { Kruskal-Wallis } \\
\text { (Teste H) }\end{array}$ & 1 & Michelino et al., (2017) \\
\hline
\end{tabular}

Fonte: Elaboraçẫo dos autores.

Sintetizando a análise dos aspectos descritivos da revisão, identificou-se, portanto, a tendência para pesquisas empíricas, baseadas em métodos quantitativos, com utilização de surveys, e técnicas de análise fundamentadas em estatística inferencial. 


\subsection{Categorização dos temas, abordagens teóricas e a identificação de fatores de desenvolvimento da ACAP para inovação em NETBs e startups}

Com a revisão da literatura, foi possível identificar um panorama sobre os estudos relativos à ACAP direcionada para inovação em NEBTs e startups. Para a categorização dos temas e abordagens teóricas buscou-se identificar a proximidade de foco de estudo entre os artigos levantados. Em seguida, os estudos foram categorizados a posteriori por agrupamentos específicos de acordo com a temática relacionada à ACAP. Adicionalmente, foram identificados fatores que influenciam positivamente o desenvolvimento da ACAP para a criação de inovação nestes empreendimentos (Quadro 3).

\subsection{Discussão, lacunas e direcionamentos para avanços teóricos}

\subsubsection{Panorama do desenvolvimento da ACAP para inovação em NEBTs e startups}

A partir dos resultados demonstrados no Quadro 3, é possível identificar insights sobre o recente panorama dos estudos da ACAP nestes empreendimentos associado ao referencial teórico levantado. Para uma melhor sistematização da discussão, são descritas a seguir tais percepções segmentadas pelas quatro categorias temáticas apresentadas: a relação de ACAP e redes, antecedentes e potencial de realização, exploração de fatores/recursos internos ou externos e estratégias de inovação.

\section{ACAP e redes}

A busca de conhecimento e complementariedades externas em redes se evidencia como fator determinante para a absorção de recursos e conhecimentos relevantes. Evidências teóricas indicam que essa busca ocorre devido à escassez e necessidade inicial de se agregar capacidades externas complementares (HYYTINEN; PAJARINEN; ROUVINEN., 2015). Tais necessidades fazem com que estes empreendimentos sejam mais dependentes de parceiros e alianças, impulsionando-os a assumir riscos associados à carência de capacidades para o desenvolvimento de inovações. Neste processo, o capital social destes empreendimentos é primordial para a obtençáo de maior capacidade absortiva se comparado aos empreendimentos tradicionais (DE JONG; FREEL, 2010, SALISU; ABU BAKAR, 2019). 
QUADRO 3

Distribuiçáo dos estudos por categorias temáticas, abordagens e agrupamento de fatores de desenvolvimento da ACAP para inovaçáo em NEBTs e startups

\begin{tabular}{|c|c|c|c|}
\hline $\begin{array}{c}\text { Categoria da } \\
\text { ACAP }\end{array}$ & Abordagens & Fatores & Autores \\
\hline \multirow{3}{*}{$\begin{array}{l}\text { ACAP e Redes } \\
36 \text { artigos } \\
(34,95 \%)\end{array}$} & $\begin{array}{l}\text { Relação entre a } \\
\text { ACAP e a rede de } \\
\text { atores externos } \\
(17 \text { artigos, } \\
16,50 \%)\end{array}$ & $\begin{array}{l}\text { - Investimentos } \\
\text { - Capacidade e suporte de P\&D } \\
\text { - Configuraçáo de redes } \\
\text { - Conhecimento prévio } \\
\text { envolvido } \\
\text { - Engajamento } \\
\text { - Portfolio de alianças e diver- } \\
\text { sificaçăo }\end{array}$ & $\begin{array}{l}\text { Benson e Ziedonis (2009); Lee, Kim e Jang (2015) } \\
\text { De Jong e Freel (2010); Faria, Lima e Santos } \\
\text { (2010); Malik e Wei (2011); Unsal e Taylor (2011) } \\
\text { Hurmelinna-Laukkanen (2012); Martínez-Cañas, } \\
\text { Sáez Martínez e Ruiz Palomino (2012); Perez, } \\
\text { Whitelock e Florin (2013) } \\
\text { Hughes et al. (2014) } \\
\text { Nambisan (2013); Patton, (2014); Grandinetti } \\
\text { (2016) } \\
\text { Díez-Vial e Montoro-Sánchez (2016); Xu (2016); } \\
\text { Garcia Martinez, Zouaghi e Sanchez Garcia (2017); } \\
\text { Wu et al. (2017) }\end{array}$ \\
\hline & $\begin{array}{l}\text { Relaçáo entre } \\
\text { a ACAP e } \\
\text { transferência de } \\
\text { conhecimento } \\
(10 \text { artigos, } \\
9,71 \%)\end{array}$ & $\begin{array}{l}\text { - Fluxos de conhecimento } \\
\text { - Transferência de tecnologia/ } \\
\text { conhecimento } \\
\text { - Capital humano e intelectual } \\
\text { - Redes informais }\end{array}$ & $\begin{array}{l}\text { Rothaermel e Thursby (2005); Lichtenthaler (2008) } \\
\text { Chan } \text { et al. (2010); Filatotchev } \text { et al. (2011); Fuku- } \\
\text { gawa (2013); Fernández-Esquinas } \text { et al. (2016) } \\
\text { Awang, Hussain e Malek (2013); Mariano e Walter } \\
\text { (2015); Engelman } \text { et al. (2017) } \\
\text { Martin-Rios e Erhardt (2016) }\end{array}$ \\
\hline & $\begin{array}{l}\text { Relação entre a } \\
\text { ACAP e a Inova- } \\
\text { çấo Aberta } \\
(9 \text { artigos, } \\
8,74 \%)\end{array}$ & $\begin{array}{l}\text { - Rotinas e práticas de Inovação } \\
\text { Aberta } \\
\text { - Escolha de parceiros e formas } \\
\text { de ligação } \\
\text { - Colaboraçōes abertas de P\&D } \\
\text { - Folga de recursos entre } \\
\text { parceiros }\end{array}$ & $\begin{array}{l}\text { Robertson, Casali e Jacobson (2012); Cheng e } \\
\text { Chen (2013); Kim, Kim e Foss (2016); Flor, } \\
\text { Cooper e Oltra (2017) } \\
\text { Van Geenhuizen e Ye (2014); Cruz-González, } \\
\text { López-Sáez e Navas-López (2015) } \\
\text { Martin-de Castro (2015); Michelino et al. (2017) } \\
\text { Wang, Guo e Yin (2017) }\end{array}$ \\
\hline \multirow{4}{*}{$\begin{array}{l}\text { ACAP, Antece- } \\
\text { dentes e } \\
\text { potencial de } \\
\text { realizaçáo }\end{array}$} & $\begin{array}{l}\text { Relação entre o } \\
\text { potencial de rea- } \\
\text { lizaçăo da ACAP } \\
\text { e seu impacto na } \\
\text { performance da } \\
\text { inovação } \\
(10 \text { artigos, } \\
9,71 \%)\end{array}$ & $\begin{array}{l}\text { - Distância cognitiva e adaptação } \\
\text { - Estruturas para internaciona- } \\
\text { lizaçấo } \\
\text { - Experiência e capacidades } \\
\text { prévias } \\
\text { - Plataformas virtuais integrativas } \\
\text { - Orientaçăo empreendedora } \\
\text { e riscos }\end{array}$ & $\begin{array}{l}\text { Nooteboom et al. (2007); Wang e Han (2011) } \\
\text { Rhee (2008) } \\
\text { Chen, Lin e Chang (2009); Zheng, Liu e George } \\
\text { (2010) } \\
\text { Teigland et al. (2014) } \\
\text { Petti e Zhang (2013); Hyytinen, Pajarinen e } \\
\text { Rouvinen (2015); Hughes et al. (2017); Larrañeta, } \\
\text { Galán González e Aguilar (2017) }\end{array}$ \\
\hline & $\begin{array}{l}\text { Relação entre } \\
\text { a ACAP e seus } \\
\text { antecedentes } \\
(7 \text { artigos, } 6,8 \%)\end{array}$ & $\begin{array}{l}\text { - Conhecimento e experimen- } \\
\text { tação } \\
\text { - Líderes mais abertos } \\
\text { - Estratégias prévias de interna- } \\
\text { cionalização } \\
\text { - Existência de ambiente incerto }\end{array}$ & $\begin{array}{l}\text { Fosfuri e Tribó (2008); Burcharth, Lettl e Ulhøi } \\
\text { (2015); Stulova e Rungi (2017); Yao e Chang } \\
\text { (2017) } \\
\text { Noblet, Simon e Parent (2011) } \\
\text { Coeurderoy et al. (2012) } \\
\text { Shoham et al. (2017) }\end{array}$ \\
\hline & $\begin{array}{l}\text { Relação entre a } \\
\text { ACAP e o grau } \\
\text { de inovaçáo } \\
(6 \text { artigos, } \\
5,83 \%)\end{array}$ & $\begin{array}{l}\text { - Recursos investidos } \\
\text { - Funcionários bem qualificados } \\
\text { - Uso de fluxos de conheci- } \\
\text { mento } \\
\text { - Capital social e organizacional } \\
\text { - Pesquisa básica } \\
\text { - Capacidade em área específica }\end{array}$ & $\begin{array}{l}\text { Deeds (2001) } \\
\text { Harison e Koski (2010) } \\
\text { Kostopoulos et al. }(2011) \\
\text { Aribi e Dupouët (2015) } \\
\text { Martinez-Senra } \text { et al. } \text { (2015) } \\
\text { Sheng e Chien (2016) }\end{array}$ \\
\hline & $\begin{array}{l}25 \text { artigos } \\
(24,27 \%)\end{array}$ & $\begin{array}{l}\text { - Agrupamento de práticas } \\
\text { focadas em orientaçáo para } \\
\text { aprendizagem, recursos, estru- } \\
\text { tura, gestão do conhecimento, e } \\
\text { experiência } \\
\text { - Agrupamento de capacidades } \\
\text { gerenciais, projetos abertos } \\
\text { para colaboração, engajamento, } \\
\text { recursos e regimes de apropria- } \\
\text { bilidade }\end{array}$ & Camisón e Forés (2010) \\
\hline
\end{tabular}


QUADRO 3

Distribuiçáo dos estudos por categorias temáticas, abordagens e agrupamento de fatores de desenvolvimento da ACAP para inovaçáo em NEBTs e startups

\begin{tabular}{|c|c|c|c|}
\hline $\begin{array}{l}\text { Categoria da } \\
\text { ACAP }\end{array}$ & Abordagens & Fatores & Autores \\
\hline \multirow{3}{*}{$\begin{array}{l}\text { ACAP e Fatores/ } \\
\text { recursos internos } \\
\text { ou externos } \\
23 \text { artigos } \\
(22,33 \%)\end{array}$} & $\begin{array}{l}\text { Relaçáo entre a } \\
\text { ACAP e exploraçấo } \\
\text { de recursos } \\
(13 \text { artigos, } 12,62 \%)\end{array}$ & $\begin{array}{l}\text { - P\&D e recursos internos } \\
\text { - Base de conhecimento e capítal } \\
\text { intelectual } \\
\text { - Capacidades organizacionais e } \\
\text { financeiras } \\
\text { - Folga de recursos } \\
\text { - Recursos complementares de co- } \\
\text { laboraçáo } \\
\text { - Turbulência de mercado e com- } \\
\text { petitividade } \\
\text { - Empreendedorismo corporativo } \\
\text { - Aquisiçóes tecnológicas }\end{array}$ & $\begin{array}{l}\text { Vega-Jurado et al. (2008); Serrano-Bedia, Ló- } \\
\text { pez-Fernández e García-Piqueres (2012); Wang } \\
\text { et al. (2016) } \\
\text { Zhou e Li (2012); Martín-de Castro et al. (2013) } \\
\text { Tavani, Sharifi e Ismail (2013); Chitsaz, Liang } \\
\text { Khoshsoroor (2017) } \\
\text { Lai e Weng (2014) } \\
\text { Eftekhari e Bogers (2015) } \\
\text { Yoo, Sawyerr e Tan (2015); Aniruddha e Mital } \\
\text { (2016) } \\
\text { García-Sánchez, García-Morales e Martín-Rojas } \\
(2017) \\
\text { Sears (2017) }\end{array}$ \\
\hline & $\begin{array}{l}\text { Relação entre a } \\
\text { ACAP e fatores } \\
\text { para produçáo da } \\
\text { inovaçáo } \\
(8 \text { artigos, } 7,77 \%)\end{array}$ & $\begin{array}{l}\text { - Processamento de fluxos de co- } \\
\text { nhecimento } \\
\text { - Interação de recursos para hete- } \\
\text { rogeneidade } \\
\text { - Competências tecnológicas e ex- } \\
\text { pertise } \\
\text { - Rotas abertas de exploraçáo cien- } \\
\text { tífica } \\
\text { - Habilidades para parceiria de } \\
\text { negócios }\end{array}$ & $\begin{array}{l}\text { Jantunen (2005) } \\
\text { Liefner, Wei e Zeng (2013) } \\
\text { García-Morales, Bolívar-Ramos e Martín-Rojas } \\
\text { (2014); Huang et al. (2015); Pereira e Leităo } \\
\text { (2016); Galbraith (2017) } \\
\text { Li, Youtie e Shapira (2015) } \\
\text { Agostini e Nosella (2017) } \\
\end{array}$ \\
\hline & $\begin{array}{l}\text { Relação entre a } \\
\text { ACAP e capacidades } \\
\text { dinâmicas e recursos } \\
(2 \text { artigos, } 1,94 \%)\end{array}$ & $\begin{array}{l}\text { - Recursos em contextos específicos } \\
\text { - Capacidades adaptativas por meio } \\
\text { de TICs }\end{array}$ & $\begin{array}{l}\text { Hullova, Trott e Don Simms (2016) } \\
\text { Parida, Oghazi e Cedergren (2016) }\end{array}$ \\
\hline \multirow{5}{*}{$\begin{array}{l}\text { ACAP e Estraté- } \\
\text { gias de inovaçáo }\end{array}$} & $\begin{array}{l}\text { Relação entre a } \\
\text { ACAP e posiciona- } \\
\text { mento estratégico } \\
\text { para inovaçáo } \\
(9 \text { artigos, } 8,74 \%)\end{array}$ & $\begin{array}{l}\text { - Estratégia de combinação de co- } \\
\text { nhecimento } \\
\text { - Desenvolvimento, exploração e } \\
\text { mercados } \\
\text { - Estratégias de aprendizagem } \\
\text { - Estratégias de exploração de C,T\&I } \\
\text { - Identificação de oportunidades }\end{array}$ & $\begin{array}{l}\text { Veugelers e Cassiman (1999); Fernhaber e Patel } \\
\text { (2012) } \\
\text { Van Geenhuizen e Nijkamp (2012); Berghman } \\
\text { et al. (2013) } \\
\text { Anatoliivna (2013) } \\
\text { Grimpe e Sofka (2009); Huang (2017); Teirlinck } \\
\text { (2017) } \\
\text { Saemundsson e Candi (2017) }\end{array}$ \\
\hline & $\begin{array}{l}\text { Relação entre a } \\
\text { ACAP e a criaçáo } \\
\text { do conhecimento } \\
\text { tecnológico } \\
\text { (3 artigos, } 2,91 \% \text { ) }\end{array}$ & $\begin{array}{l}\text { - Recursos humanos e tecnológicos } \\
\text { - Diversificaçăo tecnológica } \\
\text { - Orientaçẫo tecnológica e dina- } \\
\text { mismo }\end{array}$ & $\begin{array}{l}\text { Negassi (2004) } \\
\text { Sugheir, Phan e Hasan (2012) } \\
\text { Lichtenthaler (2016) }\end{array}$ \\
\hline & $\begin{array}{l}\text { Relaçáo entre a } \\
\text { ACAP e a cultura } \\
\text { organizacional } \\
(3 \text { artigos, } 2,91 \%)\end{array}$ & $\begin{array}{l}\text { - Diferenças culturais e integraçáo } \\
\text { social } \\
\text { - Cultura para inovação e empreen- } \\
\text { dedorismo } \\
\text { - Inteligência cultural e conhecimento }\end{array}$ & $\begin{array}{l}\text { Björkman, Stahl e Vaara (2007) } \\
\text { Ali e Park (2016) } \\
\text { Gölgeci, Swiatowiec-Szczepanska e Raczkowski } \\
\text { (2017) }\end{array}$ \\
\hline & $\begin{array}{l}\text { ACAP como prin- } \\
\text { cipal variável para o } \\
\text { desenvolvimento da } \\
\text { inovação } \\
(2 \text { artigos, } 1,94 \%)\end{array}$ & $\begin{array}{l}\text { - Ampliaçáo/profundidade de conhe- } \\
\text { cimento } \\
\text { - Atuação em setores estratégicos }\end{array}$ & $\begin{array}{l}\text { Ferreras-Méndez et al. (2015) } \\
\text { Luo et al. (2017) }\end{array}$ \\
\hline & $\begin{array}{l}\text { Relação entre a } \\
\text { ACAP e a economia } \\
\text { do conhecimento } \\
(2 \text { artigos, } 1,94 \%)\end{array}$ & $\begin{array}{l}\text { - Aprendizagem tecnológica e TICs } \\
\text { - Posiçắo estratégica e oportunidade }\end{array}$ & $\begin{array}{l}\text { Carayannis et al. (2006) } \\
\text { Mueller (2007) }\end{array}$ \\
\hline
\end{tabular}

Fonte: Elaboraçáo dos autores. 


\section{Antecedentes e potencial de realizaçáo}

As capacidades de identificação de oportunidades para obtenção e assimilação de conhecimento são elementos intrínsecos de desenvolvimento destes empreendimentos (SAEMUNDSSON; CANDI, 2017). Por meio de sua orientaçẫo empreendedora, estes negócios assumem projetos e atividades inovativas em ambientes incertos que estimulam o desenvolvimento da capacidade absortiva (COLOMBO; PIVA, 2008; COLOMBO; D’ADDA; PIVA, 2010). A ocorrência destes fatores também se evidencia pelo papel dos líderes destes empreendimentos e o capital humano existente. Os fundadores destes negócios se tornam atores do estímulo de desenvolvimento de conexóes externas para reduzir a lacuna de conhecimento e recursos existentes (DE JONG; FREEL, 2010). Adicionalmente, o capital humano, quando acompanhado de maior qualificação, se torna um fator determinante para a capacidade de absorção tecnológica destas empresas (COHEN; LEVINTHAL, 1990; KATO, 2020).

\section{Fatores/recursos internos ou externos}

A exploração e integração de recursos internos e externos potencializam as capacidades absortivas destes empreendimentos para a conversão de conhecimento em produtos e serviços comercializáveis (ZHENG; LIU; GEORGE 2010; SALISU; ABU BAKAR, 2019). O sucesso mercadológico de inovaçôes desenvolvidas por estes empreendimentos depende da disponibilidade de processos exploratórios de recursos para potencializar a ACAP (PARADKAR; KNIGHT; HANSEN, 2015). Por exemplo, a falta de recursos humanos qualificados, recursos financeiros e inexperiência em colaboraçóes podem dificultar o desenvolvimento de novos processos de inovação (SPENDER et al., 2017). Fatores relacionados à experiência anterior em colaboraçôes também contribuem para fomentar a confiança em novas alianças. Neste caso, experiência promove reputação interorganizacional, que é particularmente importante para facilitar o acesso aos recursos externos (AGGARWAL; HSU, 2009; RASMUSSEN; TANEV, 2015).

\section{Estratégias de inovação}

As NEBTs e startups enfrentam os desafios do desenvolvimento tecnológico incerto, necessitando de flexibilidade estratégica para se adaptar e sobreviver em ambientes em mudança (ROBERTS; MEYER, 1991; ANDRIES; DEBACKERE, 2007; CANDI; VAN DEN ENDE; GEMSER, 2013; SAEMUNDSSON; CANDI, 2017). Roti- 
nas, procedimentos e planejamento estratégico para inovar são, em geral, recursos benéficos para as NEBTs. Já para as startups, que possuem estruturas organizacionais mais enxutas, a flexibilidade relacionada à sua capacidade adaptativa se torna fator mais relevante. Para ambos os empreendimentos, ser competitivo requer não apenas o desenvolvimento de capacidades inovadoras internas eficazes, mas também posicionamento estratégico para acessar um conjunto diversificado de informaçôes e recursos para se manter a par das mudanças tecnológicas e institucionais (ZHENG; LIU; GEORGE, 2010).

\subsubsection{Lacunas e direcionamentos}

Além das abordagens e categorias da ACAP direcionadas para inovação, a revisão da literatura possibilitou a proposição de relevante agenda de pesquisa e direcionamentos no contexto das NEBTs e startups. As lacunas sintetizadas na Figura 1 apresentam oportunidades para investigação, avanços e caminhos sobre os quais a ACAP se desenvolve no contexto destes empreendimentos.

Com a revisão da literatura, identificou-se um conjunto de fatores facilitadores da ocorrência da ACAP direcionada para a inovação em NEBTs e startups. Entretanto, fatores ou microantecedentes vinculados ao potencial de realização da ACAP ainda carecem de modelos interativos que congreguem motivaçóes inerentes a estes empreendimentos. Futuros estudos podem propor modelos integrativos que relacionem capacidades prévias como gatilhos determinantes do processo de inovação, o papel de experiências anteriores, a path dependency em direção a um modelo de risco tecnológico, e a orientação para a aprendizagem como estratégia previamente deliberada em oposição a buscas por necessidades iniciais.

Com a identificação e assimilação de conhecimentos relevantes para o desenvolvimento de inovaçóes, os recursos internos ou externos assumem um papel essencial nos processos da ocorrência da ACAP. Comumente, as NEBTs e startups, em suas fases iniciais, buscam por investimentos e recursos que possam potencializar as capacidades de infraestrutura tecnológica e de capital humano para a criação de inovaçóes. Porém, poucos estudos foram identificados e pouco se sabe sobre o papel potencializador destes recursos na conversão da PACAP em RACAP. As especificidades dos recursos fornecidos, sua utilização e a mensuração dos resultados de inovação são promissores fatores de desenvolvimento a serem investigados por estudos empíricos relacionados à ACAP. 


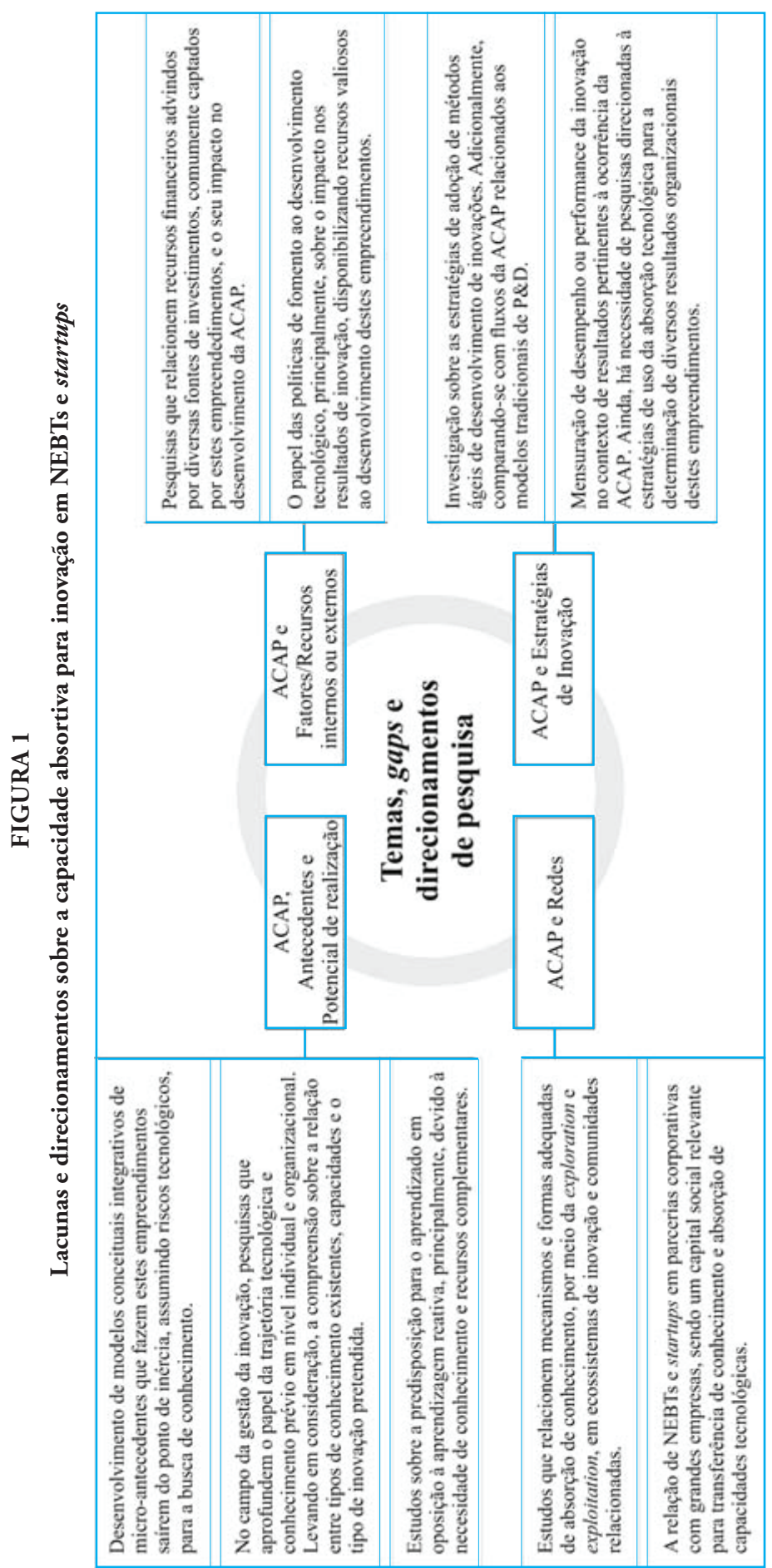


Redes e colaboraçôes externas são amplamente investigadas na literatura sobre a ACAP. Como exemplo, foram observados estudos sobre a atuação de NEBTs em ambientes de inovação relacionados a parques científicos e tecnológicos, sendo ambientes complexos e relevantes fontes de absorção de conhecimento com uso intenso de tecnologias. Entretanto, contextos específicos relacionados a outros ecossistemas e ambientes de inovação foram incipientemente explorados, como incubadoras de empresas, aceleradoras, redes e plataformas virtuais de suporte a estes empreendimentos. Da mesma forma, incluem-se programas corporativos que integram grandes empresas com estes empreendimentos na perspectiva de Inovaçáo Aberta, envolvendo transferência de conhecimento e capacidades para o desenvolvimento de inovaçóes.

Quanto às estratégias e processos de pesquisa e desenvolvimento (P\&D), a literatura existente carece de ampla investigação sobre novos métodos ágeis e enxutos adotados principalmente pelas startups. Incluem-se novos processos que se opóem a modelos tradicionais de produção, envolvendo capacidades adaptativas, desenvolvimento baseado em customer experience, e projetos com equipes reduzidas. Por fim, indica-se a proposição de estudos que investiguem as estratégias e processos decisórios destes empreendimentos quanto ao uso de capacidades tecnológicas absorvidas para a escolha e desenvolvimento de projetos tecnológicos, incluindo a mensuração da performance resultante das estratégias adotadas.

\section{Conclusão}

Partindo dos pressupostos cunhados por Cohen e Levinthal (1990), pelos quais a ACAP se relaciona com as habilidades de reconhecimento, assimilação de conhecimento para aplicação mercadológica como fator crítico para a criação de inovação, se faz necessário o avanço de estudos teórico-empíricos para a compreensão de processos e identificação de como a ACAP ocorre em novos empreendimentos focados em tecnologia.

Neste sentido, o presente estudo estruturou o conhecimento sobre a ACAP sistematizando a literatura sobre o tema relacionado à inovação em novas empresas de base tecnológica e startups. Adicionalmente, a presente pesquisa identificou temas, abordagens e um extenso conjunto de fatores de desenvolvimento da ACAP para inovação nestes empreendimentos.

As abordagens da ACAP aqui analisadas permitiram observar a evolução do construto, antes caracterizada pela forte vinculação a atividades de P\&D (LANE; KOKA; PATHAK, 2006) para uma perspectiva mais ampla envolvendo redes, 
antecedentes, potencial de realização, exploração de fatores/recursos diversos e estratégias de inovação.

Logo, até que ponto a ACAP e suas dimensóes existentes demandariam uma reconfiguração para modelos mais atuais baseados em avanços tecnológicos para as diferentes vertentes da inovação? $\mathrm{Ou}$, como as novas empresas de base tecnológica e startups podem potencializar e realizar a ACAP diferentemente de outras tipologias de empresas? Este trabalho náo pretendeu, per se, responder profundamente tais indagaçóes, mas pode contribuir para futuras respostas com o panorama de estudos de alta qualidade apresentado.

A distribuição de enquadramento das quatro categorias e abordagens da ACAP, e mais relevante, com o conjunto de fatores de desenvolvimento identificados, possibilita o despertar para um futuro esboço de agenda de pesquisa. De forma complementar, os fatores identificados contribuem para a proposição de modelos conceituais, escalas e investigação da ocorrência da ACAP para avanços no contexto específico destes empreendimentos.

Como limitação, importa destacar que este trabalho se ateve à revisão e análise do qualificado material levantado com respectivas abordagens teóricas, fatores da ACAP para inovação e discussão das lacunas existentes. Não foi explorada, por exemplo, a existência de modelos conceituais observados nos artigos da revisão da literatura. O detalhamento da integração de componentes-chave, ou variáveis que estão condicionadas a esses modelos podem contribuir para a compreensão de como as NEBTs e startups absorvem conhecimento para o alcance do desenvolvimento tecnológico.

Futuros estudos também são encorajados a explorar possíveis diferenciações da ocorrência da ACAP entre as tipologias dos empreendimentos investigados, principalmente, nas startups, como um fenômeno recente ainda a ser amplamente explorado. Há também a limitação deste estudo e a possibilidade de futuras pesquisas considerarem outras tipologias de firmas e não exclusivamente NEBTs e startups, como por exemplo, outras empresas que se enquadrem como technology companies ou technology firms que podem não se caracterizar necessariamente ao contexto dos empreendimentos investigados neste estudo.

Como dito anteriormente, este estudo abre espaço para uma possível agenda na temática. Foram identificadas lacunas e providos direcionamentos relevantes para agendas propositivas, trazendo alguns esboços representados nas quatro categorias temáticas e fatores de desenvolvimento da ACAP que possibilitam ampla investigação sobre a inovação nestes empreendimentos. 


\section{Agradecimentos:}

Fundação de Apoio à Pesquisa do Distrito Federal (FAP/DF).

\section{Referências}

AGGARWAL, V.A.; HSU, D.H. Modes of cooperative R\&D commercialization by startups. Strategic Management Journal, v. 30, n. 8, p. 835-864, 2009.

AGOSTINI, L.; NOSELLA, A. A dual knowledge perspective on the determinants of SME patenting: results of an empirical investigation. Management Decision, v. 55, n. 6, p. 1226-1247, 2017.

ALI, M.; PARK, K. The mediating role of an innovative culture in the relationship between absorptive capacity and technical and non-technical innovation. Journal of Business Research, v. 69, n. 5, p. 1669-1675, 2016.

ANATOLIIVNA, V. O. Absorptive capacity in organizational theories: Learning, innovation, managerial cognition. Marketing \& Management of Innovations, v. 4, p. 190-199, 2013.

ANDRIES, P.; DEBACKERE, K. Adaptation and performance in new businesses: Understanding the moderating effects of independence and industry. Small business economics, v. 29, n. 1-2, p. 81-99, 2007.

ANIRUDDHA, K., MITAL, A. Role of Dynamic Capabilities in Innovation Output of High-Technology Firms. Strategic Change, v. 25, n. 4, p. 401-425, 2016.

ARIBI, A.; DUPOUËT, O. The role of organizational and social capital in the firm's absorptive capacity. Journal of Knowledge Management, v. 19, n. 5, p. 987-1006, 2015.

ARTHUR D. LITTLE LTD.; ANGLO-GERMAN FOUNDATION FOR THE STUDY OF INDUSTRIAL SOCIETY. New technology-based firms in the United Kingdom and the Federal Republic of Germany: a report. London: Wilton House Publications, 1977.

AWANG, A.H.; HUSSAIN, M.Y.; MALEK, J.A. Knowledge transfer and the role of local absorptive capability at science and technology parks. The Learning Organization, v. 20, n. 4/5, p. 291-307, 2013.

BENSON, D.; ZIEDONIS, R.H. Corporate Venture Capital as a Window on New Technologies: Implications for the Performance of Corporate Investors When Acquiring Start-ups. Organization Science, v. 20, n. 2, p. 329-351, 2009.

BERGHMAN, L.; MATTHYSSENS, P.; STREUKENS, S.; VANDENBEMPT, K. Deliberate Learning Mechanisms for Stimulating Strategic Innovation Capacity. Long Range Planning, v. 46, n. 1, p. 39-71, 2013. 
BJÖRKMAN, I.; STAHL, G.; VAARA, E. Cultural differences and capability transfer in crossborder acquisitions: the mediating roles of capability complementarity, absorptive capacity, and social integration. Journal of International Business Studies, v. 38, n. 4, p. 658-672, 2007. BLANK, S.; DORFF, B. The start-up owner's manual: The step-by-step guide for building a great company. California: Book Baby, 2012.

BURCHARTH, A.L.L.A.; LETTL, C.; ULHØI, J.P. Extending organizational antecedents of absorptive capacity: Organizational characteristics that encourage experimentation. Technological Forecasting and Social Change, v. 90, p. 269-284, 2015.

CAMISÓN, C.; FORÉS, B. Knowledge absorptive capacity: New insights for its conceptualization and measurement. Journal of Business Research, v. 63, n. 7, p. 707-715, 2010.

CANDI, M.; VAN DEN ENDE, J.; GEMSER, G. Organizing innovation projects under technological turbulence. Technovation, v. 33, n. 4, p. 133-141, 2013.

CARAYANNIS, E. G.; POPESCU, D.; SIPP, C.; STEWART, M. Technological learning for entrepreneurial development (TL4ED) in the knowledge economy (KE): Case studies and lessons learned. Technovation, v. 26, n. 4, p. 419-443, 2006.

CHAN, K.A.; OERLEMANS, L.A.G.; PRETORIUS, M.W. Knowledge exchange behaviours of science park firms: the innovation hub case. Technology Analysis \& Strategic Management, v. 22, n. 2, p. 207-228, 2010.

CHEN, Y.S.; LIN, M.J.J.; CHANG, C.H. The positive effects of relationship learning and absorptive capacity on innovation performance and competitive advantage in industrial markets. Industrial Marketing Management, v. 38, n. 2, p. 152-158, 2009.

CHENG, C.C.J.; CHEN, J. Breakthrough innovation: the roles of dynamic innovation capabilities and open innovation activities. Journal of Business \& Industrial Marketing, v. 28, n. 5, p.444-454, 2013.

CHITSAZ, E.; LIANG, D.; KHOSHSOROOR, S. The impact of resource configuration on Iranian technology venture performance. Technological Forecasting and Social Change, v. 122, p. 186-195, 2017.

COEUrderoy, R.; COWLING, M.; LiCHT, G.; MURRAY, G. Young firm internationalization and survival: Empirical tests on a panel of 'adolescent' new technologybased firms in Germany and the UK. International Small Business Journal, v. 30, n. 5, p. 472-492, 2012.

COHEN, W.; LEVINTHAL, D. Innovation and Learning: The Two Faces of R \& D. The Economic Journal, v. 99, n. 397, p. 569-596, 1989. 
COHEN, W.; LEVINTHAL, D. Absorptive Capacity: A New Perspective on Learning and Innovation. Administrative Science Quarterly, v. 35, n. 1, p. 128-152, 1990.

COLOMBO, M.G.; PIVA, E. Strengths and weaknesses of academic start-ups: A conceptual model. IEEE Transactions on Engineering Management, v. 55, n. 1, p. 37-49, 2008.

COLOMBO, M.G.; D’ADDA, D.; PIVA, E. The contribution of university research to the growth of academic start-ups: An empirical analysis. The Journal of Technology Transfer, v. 35, n. 1, p. 113-140, 2010.

CRUZ-GONZÁLEZ, J.; LÓPEZ-SÁEZ, P.; NAVAS-LÓPEZ, J. E. Absorbing knowledge from supply-chain, industry and science: The distinct moderating role of formal liaison devices on new product development and novelty. Industrial Marketing Management, v. 47, p. $75-85,2015$.

DE JONG, J.P.J.; FREEL, M. Absorptive capacity and the reach of collaboration in high technology small firms. Research Policy, v. 39, n. 1, p. 47-54, 2010.

DEEDS, D.L. The role of R\&D intensity, technical development and absorptive capacity in creating entrepreneurial wealth in high technology start-ups. Journal of Engineering and Technology Management, v. 18, n. 1, p. 29-47, 2001.

DESCHESNES, M.; DROUIN, N.; COUTURIER, Y. Schools' absorptive capacity to innovate in health promotion. Journal of Health Organization and Management, v. 27, n. 1, p. 24-41, 2013.

DÍEZ-VIAL, I.; MONTORO-SÁNCHEZ, A. How knowledge links with universities may foster innovation: The case of a science park. Technovation, v. 50-51, p. 41-52, 2016.

EFTEKHARI, N.; BOGERS, M. Open for Entrepreneurship: How Open Innovation Can Foster New Venture Creation. Creativity and Innovation Management, v. 24, n. 4, p. 574-584, 2015.

EISENHARDT, K.M.; MARTIN, J.A. Dynamic capabilities: what are they? Strategic Management Journal, v. 21, n. 10/11, p. 1105-1121, 2000.

ENGELMAN, R.M.; FRACASSO, E.M.; SCHMIDT, S.; ZEN, A.C. Intellectual capital, absorptive capacity and product innovation. Management Decision, v. 55, n. 3, p. 474-490, 2017.

FARIA, P.; LIMA, F; SANTOS, R. Cooperation in innovation activities: The importance of partners. Research Policy, v. 39, n. 8, p. 1082-1092, 2010.

FERNÁNDEZ-ESQUINAS, M.; PINTO, H.; YRUELA, M.P.; PEREIRA, T. S. Tracing the flows of knowledge transfer: Latent dimensions and determinants of university-industry 
interactions in peripheral innovation systems. Technological Forecasting and Social Change, v. 113, p. 266-279, 2016.

FERNHABER, S.A.; PATEL, P.C. How do young firms manage product portfolio complexity? The role of absorptive capacity and ambidexterity. Strategic Management Journal, v. 33, n. 13, p. 1516-1539, 2012.

FERRERAS-MÉNDEZ, J. L.; NEWELL, S.; FERNÁNDEZ-MESA, A.; ALEGRE, J. Depth and breadth of external knowledge search and performance: The mediating role of absorptive capacity. Industrial Marketing Management, v. 47, p. 86-97, 2015.

FERTÖ, I; MOLNÁR, A.; TÓTH, J. Borderless ideas - open innovation in the Hungarian food chain. British Food Journal, v. 118, n. 6, p. 1494-1515, 2016.

FILATOTCHEV, I.; LIU, X.; LU, J.; WRIGHT, M. Knowledge spillovers through human mobility across national borders: Evidence from Zhongguancun Science Park in China. Research Policy, v. 40, n. 3, p. 453-462, 2011.

FLATTEN, T.C.; ENGELEN, A.; ZAHRA, S.A.; BRETTEL, M. A measure of absorptive capacity: Scale development and validation. European Management Journal, v. 29, n. 2, p. 98-116, 2011.

FLOR, M.L.; COOPER, S.Y.; OLTRA, M.J. External knowledge search, absorptive capacity and radical innovation in high-technology firms. European Management Journal, v. 36, n. 2, p. 183-194, 2017.

FOSFURI, A.; TRIBÓ, J.A. Exploring the antecedents of potential absorptive capacity and its impact on innovation performance. Omega, v. 36, n. 2, p. 173-187, 2008.

FUKUGAWA, N. University spillovers into small technology-based firms: channel, mechanism, and geography. Journal of Technology Transfer, v. 38, n. 4, p. 415-431, 2013.

GALBRAITH, B.; MCADAM, R.; WOODS, J.; MCGOWAN, T. Putting policy into practice: an exploratory study of SME innovation support in a peripheral UK region. Entrepreneurship and Regional Development, v. 29, n. 7-8, p. 668-691, 2017.

GARCIA MARTINEZ, M.; ZOUAGHI, F.; SANCHEZ GARCIA, M. Capturing value from alliance portfolio diversity: The mediating role of $P \& D$ human capital in high and low-tech industries. Technovation, v. 59, p. 55-67, 2017.

GARCÍA-MORALES, V.J.; BOLÍVAR-RAMOS, M.T.; MARTÍN-ROJAS, R. Technological variables and absorptive capacity's influence on performance through corporate entrepreneurship. Journal of Business Research, v. 67, n. 7, p. 1468-1477, 2014.

GARCÍA-SÁNCHEZ, E.; GARCÍA-MORALES, V.J.; MARTÍN-ROJAS, R. Analysis of the influence of the environment, stakeholder integration capability, absorptive capacity, 
and technological skills on organizational performance through corporate entrepreneurship. International Entrepreneurship and Management Journal, p. 1-33, 2017.

GEBAUER, H.; WORCH, H. Absorptive Capacity (of Organizations). In: WRIGHT, J.D. (ed.). International Encyclopedia of the Social and Behavioral Sciences, 2015. p. 12-19.

GÖLGECI, I.; SWIATOWIEC-SZCZEPANSKA, J.; RACZKOWSKI, K. How does cultural intelligence influence the relationships between potential and realised absorptive capacity and innovativeness? Evidence from Poland. Technology Analysis \& Strategic Management, v. 29, n. 8, p. 857-871, 2017.

GRANDINETTI, R. Absorptive capacity and knowledge management in small and medium enterprises. Knowledge Management Research \& Practice, v. 14, n. 2, p. 159-168, 2016.

GRIMPE, C.; SOFKA, W. Search patterns and absorptive capacity: Low- and high-technology sectors in European countries. Research Policy, v. 38, n. 3, p. 495-506, 2009.

HARISON, E.; KOSKI, H. Applying open innovation in business strategies: Evidence from Finnish software firms. Research Policy, v. 39, n. 3, p. 351-359, 2010.

HUANG, K.G. Uncertain intellectual property conditions and knowledge appropriation strategies: Evidence from the genomics industry. Industrial and Corporate Change, v. 26, n. 1, p. 41-71, 2017.

HUANG, K.; LIN, K.; WU, H; YU, P. Absorptive capacity and autonomous R\&D climate roles in firm innovation. Journal of Business Research, v. 68, n. 1, p. 87-94, 2015.

HUGHES, M.; MORGAN, R. E.; IRELAND, R. D.; HUGHES, P. Social Capital and Learning Advantages: A Problem of Absorptive Capacity. Strategic Entrepreneurship Journal, v. 8, n. 3, p. 214-233, 2014.

HUGHES, P.; HODGKINSON, I.R.; HUGHES, M; ARSHAD, D. Explaining the entrepreneurial orientation-performance relationship in emerging economies: The intermediate roles of absorptive capacity and improvisation. Asia Pacific Journal of Management, p. 1-29, 2017.

HULLOVA, D.; TROTT, P.; DON SIMMS, C. Uncovering the reciprocal complementarity between product and process innovation. Research Policy, v. 45, n. 5, p. 929-940, 2016.

HURMELINNA-LAUKKANEN, P. Constituents and outcomes of absorptive capacity appropriability regime changing the game. Management Decision, v. 50, n. 7, p. 1178-1199, 2012.

HYYTINEN, A.; PAJARINEN, M.; ROUVINEN, P. Does innovativeness reduce start-up survival rates? Journal of Business Venturing, v. 30, n. 4, p. 564-581, 2015. 
JANTUNEN, A. Knowledge-processing capabilities and innovative performance: an empirical study. European Journal of Innovation Management, v. 8, n. 3, p. 336-349, 2005.

KATO, M. Founders' human capital and external knowledge sourcing: Exploring the absorptive capacity of start-up firms. Economics of Innovation and New Technology, v. 29, n. 2, p.184-205, 2020.

KIM, B.; KIM, E.; FOSS, N.J. Balancing absorptive capacity and inbound open innovation for sustained innovative performance: An attention-based view. European Management Journal, v. 34, n. 1, p. 80-90, 2016.

KOSTOPOULOS, K.; PAPALEXANDRIS, A.; PAPACHRONI, M; IOANNOU, G. Absorptive capacity, innovation, and financial performance. Journal of Business Research, v. 64, n. 12, p. 1335-1343, 2011.

LAI, H. C.; WENG, C.S. Accessing External Technological Knowledge for Technological Development: When Technological Knowledge Distance Meets Slack Resources. IEEE Transactions on Engineering Management, v. 61, n. 1, p. 80-89, 2014.

LANE, P.J.; KOKA, B.R.; PATHAK, S. The Reification of Absorptive Capacity: A Critical Review and Rejuvenation of the Construct. The Academy of Management Review, v. 31, n. 4, p. 833-863, 2006.

LARRAÑETA, B.; GALÁN GONZÁLEZ, J.L.; AGUILAR, R. Early efforts to develop absorptive capacity and their performance implications: differences among corporate and independent ventures. The Journal of Technology Transfer, v. 42, n. 3, p. 485-509, 2017.

LEE, S.M.; KIM, T.; JANG, S.H. Inter-organizational knowledge transfer through corporate venture capital investment. Management Decision, v. 53, n. 7, p. 1601-1618, 2015.

LEWIN, A.Y.; MASSINI, S.; PEETERS, C. Microfoundations of Internal and External Absorptive Capacity Routines. Organization Science, v. 22, n. 1, p. 81-98, 2011.

LI, Y., YOUTIE, J.; SHAPIRA, P. Why do technology firms publish scientific papers? The strategic use of science by small and midsize enterprises in nanotechnology. Journal of Technology Transfer, v. 40, n. 6, p. 1016-1033, 2015.

LICHTENTHALER, U. Relative capacity: Retaining knowledge outside a firm's boundaries. Journal of Engineering and Technology Management, v. 25, n. 3, p. 200-212, 2008.

LICHTENTHALER, U. Determinants of absorptive capacity: the value of technology and market orientation for external knowledge acquisition. Journal of Business \& Industrial Marketing, v. 31, n. 5, p. 600-610, 2016.

LIEFNER, I.; WEI, Y.D.; ZENG, G. The Innovativeness and Heterogeneity of ForeignInvested High-Tech Companies in Shanghai. Growth \& Change, v. 44, n. 3, p. 522-549, 2013. 
LUO, B.N.; LUI, S.S; KIM, Y. Revisiting the relationship between knowledge search breadth and firm innovation: A knowledge transfer perspective. Management Decision, v. 55, n. 1, p. 2-14.

MALIK, K.; WEI, J. How external partnering enhances innovation: evidence from Chinese technology-based SMEs. Technology Analysis \& Strategic Management, v. 23, n. 4, p. 401413, 2011.

MARIANO, S.; WALTER, C. The construct of absorptive capacity in knowledge management and intellectual capital research: Content and text analyses. Journal of Knowledge Management, v. 19 , n. 2, 372-400, 2015.

MARTÍN-DE CASTRO, G. Knowledge management and innovation in knowledge-based and high-tech industrial markets: The role of openness and absorptive capacity. Industrial Marketing Management, v. 47, p. 143-146, 2015.

MARTÍN-DE CASTRO, G.; DELGADO-VERDE, M.; AMORES-SALVADÓ, J.; NAVAS-LÓPEZ, J.E. Linking human, technological, and relational assets to technological innovation: exploring a new approach. Knowledge Management Research \& Practice, v. 11, n. 2, p. 123-132, 2013.

MARTÍNEZ-CAÑAS, R.; SÁEZ MARTÍNEZ, F.J.; RUIZ PALOMINO, P. Knowledge acquisition's mediation of social capital-firm innovation. Journal of Knowledge Management, v. 16, n. 1, p. 61-76, 2012.

MARTINEZ-SENRA, A.I.; QUINTAS, M.A.; SARTAL, A.; VAZQUEZ, X.H. How Can Firms' Basic Research Turn Into Product Innovation? The Role of Absorptive Capacity and Industry Appropriability. IEEE Transactions on Engineering Management, v. 62, n. 2, p. 205-216, 2015.

MARTIN-RIOS, C.; ERHARDT, N. Small business activity and knowledge exchange in informal interfirm networks. International Small Business Journal, v. 35, n. 3, p. 285-305, 2016.

MICHELINO, F.; CAMMARANO, A.; LAMBERTI, E.; CAPUTO, M. Open innovation for start-ups: A patent-based analysis of bio-pharmaceutical firms at the knowledge domain level. European Journal of Innovation Management, v. 20, n. 1, p. 112-134, 2007.

MUELLER, P. Exploiting entrepreneurial opportunities: The impact of entrepreneurship on growth. Small Business Economics, v. 28, n. 4, p. 355-362, 2007.

NAMBISAN, S. Industry technical committees, technological distance, and innovation performance. Research Policy, v. 42, n. 4, p. 928-940, 2013.

NÄTTI, S.; HURMELINNA-LAUKKANEN, P.; JOHNSTON., W. J. Absorptive capacity and network orchestration in innovation communities - promoting service innovation. Journal of Business \& Industrial Marketing, v. 29, n. 2, p.173-184, 2014. 
NEGASSI, S. R\&D co-operation and innovation a microeconometric study on French firms. Research Policy, v. 33, n. 3, p. 365-384, 2004.

NOBLET, J. P.; SIMON, E; PARENT, R. Absorptive capacity: a proposed operationalization. Knowledge Management Research \& Practice, v. 9, n. 4, p. 367-377, 2011.

NOOTEBOOM, B.; VAN HAVERBEKE, W.; DUYSTERS, G.; GILSING, V.; VAN DEN OORD, A. Optimal cognitive distance and absorptive capacity. Research Policy, v. 36, n. 7, p. 1016-1034, 2007.

PARADKAR, A.; KNIGHT, J.; HANSEN, P.C. Innovation in start-ups: Ideas filling the void or ideas devoid of resources and capabilities? Technovation, v. 41-42, p. 1-10, 2015.

PARIDA, V.; OGHAZI, P.; CEDERGREN, S. A study of how ICT capabilities can influence dynamic capabilities. Journal of Enterprise Information Management, v. 29, n. 2, p. 179-201, 2016.

PATTON, D. Realising potential: The impact of business incubation on the absorptive capacity of new technology-based firms. International Small Business Journal, v. 32, n. 8, p. $897-917,2014$.

PEREIRA, D.; LEITÃO, J. Absorptive capacity, coopetition and generation of product innovation: contrasting Italian and Portuguese manufacturing firms. International Journal of Technology Management, v. 71, n. 1-2, p. 10-37, 2016.

PEREZ, L.; WHITELOCK, J.; FLORIN, J. Learning about customers: Managing B2B alliances between small technology start-ups and industry leaders. European Journal of Marketing, n. 47, v. 3-4, p. 431-462, 2013.

PETTI, C.; ZHANG, S. Technological entrepreneurship and absorptive capacity in Guangdong technology firms. Measuring Business Excellence, v. 17, n. 2, p. 61-71, 2013.

PRESUTTI, M.; BOARI, C.; MAJOCCHI, A. The Importance of Proximity for the Start-Ups' Knowledge Acquisition and Exploitation. Journal of Small Business Management, v. 49, n. 3, p. 361-389, 2011.

RASMUSSEN, S.E.; TANEV, S. The emergence of the lean global startup as a new type of firm. Technology Innovation Management Review, v. 5, n. 11, p. 12-19, 2015.

RHEE, J.H. International expansion strategies of Korean venture firms: Entry mode choice and performance. Asian Business \& Management, v. 7, n. 1, p. 95-114, 2008.

RIES, E. The lean start-up: How today's entrepreneurs use continuous innovation to create radically successful businesses. Crown Business: New York, 2011.

ROBERTS, E.B.; MEYER, M.H. Product strategy and corporate success. IEEE Engineering Management Review, v. 19, n. 1, p. 4-18, 1991. 
ROBERTSON, P.L.; CASALI, G.L.; JACOBSON, D. Managing open incremental process innovation: Absorptive Capacity and distributed learning. Research Policy, v. 41, n. 5, p. 822-832, 2012.

ROSsetTO, C.; CARVAlHO, C.; FERREIRA, G.; PERY, C. Absorptive capacity: the role of external knowledge in organizational strategy. Revista de Administração Mackenzie, v. 20, n. 6, 2019.

ROTHAERMEL, F.T.; THURSBY, M. University-incubator firm knowledge flows: assessing their impact on incubator firm performance. Research Policy, v. 34, n. 3, p. 305-320, 2005.

SAEMUNDSSON, R.J.; CANDI, M. Absorptive capacity and the identification of opportunities in new technology-based firms. Technovation, v. 64-65, p. 43-49, 2017.

SALISU, Y.; ABU BAKAR, L.J. Technological capability, relational capability and firms' performance: The role of learning capability. Revista de Gestão, v. 27, n. 1, p. 79-99, 2019.

SEARS, J.B. When are acquired technological capabilities complements rather than substitutes? A study on value creation. Journal of Business Research, v. 78, p. 33-42, 2017.

SERRANO-BEDIA, A.M.; LÓPEZ-FERNÁNDEZ, M.C.; GARCÍA-PIQUERES, G. Complementarity between innovation activities and innovation performance: Evidence from Spanish innovative firms. Journal of Manufacturing Technology Management, v. 23, n. 5, p. 557-577, 2012.

SHENG, M.L.; CHIEN, I. Rethinking organizational learning orientation on radical and incremental innovation in high-tech firms. Journal of Business Research, v. 69, n. 6, p. 2302-2308, 2016.

SHOHAM, A.; ASSERAF, Y.; LEV, S.; FIEGENBAUM, A. Marketing and Technological Absorptive Capacities: Environmental Antecedents and Performance Outcomes in High-Tech Firms. Journal of Business-To-Business Marketing, v. 24, n. 3, p. 165-182, 2017.

SPENDER, J.; CORVELLO, V.; GRIMALDI, M.; RIPPA, P. Startups and open innovation: A review of the literature. European Journal of Innovation Management, v. 20, n. 1, p. 4-30, 2017. STOREY, D.J.; TETHER, B.S. Public policy measures to support new technology-based firms in the Europe Union. Research Policy, v. 26, n. 9, p. 1037-1057, 1998.

STULOVA, V.; RUNGI, M. Untangling the mystery of absorptive capacity: A process or a set of success factors? The Journal of High Technology Management Research, v. 28, n. 1, p. 110-123, 2017.

SUGHEIR, J.; PHAN, P.H.; HASAN, I. Diversification and Innovation Revisited: An Absorptive Capacity View of Technological Knowledge Creation. IEEE Transactions on Engineering Management, v. 59, n. 4, p. 530-539, 2012. 
TAVANI, S.N.; SHARIFI, H.; ISMAIL, H.S. A study of contingency relationships between supplier involvement, absorptive capacity and agile product innovation. International Journal of Operations \& Production Management, v. 34, n. 1, p. 65-92, 2013.

TEIGLAND, R.; GANGI, P.M.; FLÅTEN, B.; GIOVACCHINI, B.; PASTORINO, N. Balancing on a tightrope: Managing the boundaries of a firm-sponsored OSS community and its impact on innovation and absorptive capacity. Information and Organization, v. 24, n. 1, p. 25-47, 2014.

TEIRLINCK, P. Configurations of strategic P\&D decisions and financial performance in small-sized and medium-sized firms. Journal of Business Research, v. 74, p. 55-65, 2017.

UNSAL, H.I.; TAYLOR, J.E. Absorptive Capacity of Project Networks. Journal of Construction Engineering \& Management, v. 137, n. 11, p. 994-1002, 2011.

VAN GEENHUIZEN, M.; NIJKAMP, P. Knowledge virtualization and local connectedness among young globalized high-tech companies. Technological Forecasting and Social Change, v. 79, n. 7, p. 1179-1191, 2012.

VAN GEENHUIZEN, M.; YE, Q. Responsible innovators: open networks on the way to sustainability transitions. Technological Forecasting and Social Change, v. 87, p. 28-40, 2014.

VEGA-JURADO, J.; GUTIÉRREZ-GRACIA, A.; FERNÁNDEZ-DE-LUCIO, I.; MANJARRÉS-HENRÍQUEZ, L. The effect of external and internal factors on firms' product innovation. Research Policy, v. 37, n. 4, p. 616-632, 2008.

VEUGELERS, R.; CASSIMAN, B. Make and buy in innovation strategies: evidence from Belgian manufacturing firms. Research Policy, v. 28, n. 1, p. 63-80, 1999.

WANG, C.; HAN, Y. Linking properties of knowledge with innovation performance: the moderate role of absorptive capacity. Journal of Knowledge Management, v. 15, n. 5, p. 802-819, 2011.

WANG, Y.; GUO, B.; YIN, Y. Open innovation search in manufacturing firms: the role of organizational slack and absorptive capacity. Journal of Knowledge Management, v. 21, n. 3, p. 656-674, 2017.

WANG, Z.; WANG, Q.; ZHAO, X.; LYLES, M.A.; ZHU, G. Interactive effects of external knowledge sources and internal resources on the innovation capability of Chinese manufacturers. Industrial Management \& Data Systems, v. 116, n. 8, p. 1617-1635, 2016.

WU, J.; YE, R.M.; DING, L.; LU, C.; EUWEMA, M. From "transplant with the soil" toward the establishment of the innovation ecosystem: A case study of a leading high-tech company in China. Technological Forecasting and Social Change, v. 236, p. 222-234, 2017. 
XU, Y. Entrepreneurial social capital, cognitive orientation and new venture innovation. Management Research Review, v. 39, n. 5, p. 498-520, 2016.

YAO, F.K.; CHANG, S. Do Individual Employees' Learning Goal Orientation and Civic Virtue Matter? A Micro-Foundations Perspective on Firm Absorptive Capacity. Strategic Managemet Journal, v. 38, n. 10, p. 2041-2060, 2017.

YOO, S.; SAWYERR, O.; TAN, W. The impact of exogenous and endogenous factors on external knowledge sourcing for innovation: The dual effects of the external environment. The Journal of High Technology Management Research, v. 26, n. 1, p. 14-26, 2015.

ZAHRA S.A.; GEORGE G. Absorptive capacity: a review, reconceptualization, and extension. Academy of Management Review, v. 27, n. 2, p. 185-203, 2002.

ZHENG, Y.; LIU, J.; GEORGE, G. The dynamic impact of innovative capability and inter-firm network on firm valuation: A longitudinal study of biotechnology start-ups. Journal of Business Venturing, v. 25, n. 6, p. 593-609, 2010.

ZHOU, K.Z.; LI, C.B. How knowledge affects radical innovation: Knowledge base, market knowledge acquisition, and internal knowledge sharing. Strategic Management Journal, v. 33, n. 9, p. 1090-1102, 2012.

\section{Contribuiçáo dos autores}

A. Fundamentação teórico-conceitual e problematização: Bruno Alencar Pereira, Josivania Silva Farias

B. Pesquisa de dados e análise estatística: Bruno Alencar Pereira

C. Elaboração de figuras e tabelas: Bruno Alencar Pereira, Josivania Silva Farias

D. Elaboração e redação do texto: Bruno Alencar Pereira, Josivania Silva Farias

E. Seleção das referências bibliográficas: Bruno Alencar Pereira, Josivania Silva Farias

Conflito de interesse: os autores declaram não haver conflito de interesse.

Fonte de financiamento: os autores declararam não haver fonte de financiamento.

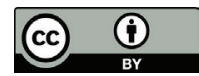

Este é um artigo publicado em acesso aberto (Open Access) sob a licença Creative Commons Attribution CC-BY, que permite uso, distribuição e reprodução em qualquer meio, sem restriçóes desde que o trabalho original seja corretamente citado. 